\title{
AVALIAÇÃO DA PRÁTICA DE ENFERMAGEM NA SEGURANÇA DO PACIENTE ONCOLÓGICO
}

Sara Maria da Costa Negrão ${ }^{1}$ Mayara Nicodemos da Conceição ${ }^{1}$ Manoel Junior Ferreira Mendes ${ }^{1}$ Jeferson Santos Araújo ${ }^{2}$

Ingrid Magali Souza Pimentel ${ }^{2}$ Mary Elizabeth de Santana' https://orcid.org/0000-0002-7828-5654

https://orcid.org/0000-0002-0142-3457 https://orcid.org/0000-0001-5778-0579 https://orcid.org/0000-0003-3311-8446 https://orcid.org/0000-0003-1820-5496 https://orcid.org/0000-0002-3629-8932

Objetivo: Avaliar as práticas adotadas por enfermeiros para a segurança dos pacientes oncológicos. Metodologia: Estudo exploratório descritivo de abordagem quantitativa, utilizando questionário "Pesquisa sobre Segurança do Paciente em Hospitais" (HSOPSC) da "Agency Healthcare Research and Quality". A pesquisa foi realizada em um hospital público de referência em atendimento oncológico em Belém-Pará. Resultados: Foram entrevistados 51 profissionais de enfermagem, sendo que 4 (7,9\%) dos profissionais relataram de 11 a 20 eventos que prejudicaram, de forma direta ou indireta, o bem-estar do paciente oncológico. Discussão: É possível detectar contradições nas respostas dos profissionais ao realizar a análise das variáveis abordadas na pesquisa. Além disso, não houve relato das ações adotadas pela enfermagem após a notificação de cada adversidade. Conclusão: A cultura de segurança ainda não se encontra no padrão ideal, sendo necessária a realização de educação permanente e avaliação frequente dos serviços prestados referentes à segurança do paciente oncológico.

Descritores: Segurança do Paciente; Enfermagem; Câncer.

\section{NURSING EVALUATION ON THE SAFETY OF THE ONCOLOGICAL}

Objective: To evaluate the practices adopted by nurses for the safety of oncological patients. Methodology: Descriptive exploratory study of quantitative approach using the "Hospital Patient Safety Survey" (HSOPSC) "questionnaire from the Healthcare Research and Quality Agency". The research was conducted at a public referral hospital for cancer treatment in Belém-Pará. Results: 51 nursing professionals were interviewed, of which 4 (7.9\%) reported 11 to 20 events that directly or indirectly affected the well-being of cancer patients. Discussion: It is possible to detect contradictions in the answers of the professionals when performing the analysis of the variables addressed in the research. Moreover, there is no report of the actions adopted by the nursing after each notification. Conclusion: The safety culture is not yet in the ideal standard, being necessary the accomplishment of permanent education and frequent evaluation of the services provided related to the cancer patient safety.

Descriptores: Patient Safety; Nursing; Câncer.

\section{EVALUACIÓN DE ENFERMERÍA SOBRE LA SEGURIDAD DEL PACIENTE ONCOLÓGICO}

Objetivo: Evaluar las prácticas adoptadas por las enfermeras para la seguridad de los pacientes con cáncer. Metodología: Estudio exploratorio descriptivo con enfoque cuantitativo que utiliza la Encuesta de Investigación y Calidad de la Salud. La investigación se realizó en un hospital público de referencia para el tratamiento del cáncer en Belém-Pará. Resultados: Se entrevistó a 51 profesionales de enfermería y 7,9\% de los profesionales informaron de 11 a 20 eventos que afectaron el bienestar del paciente con cáncer. Discusión: Es posible detectar contradicciones en las respuestas de los profesionales al analizar las variables abordadas en la investigación. Además, no hubo informes de acciones tomadas por la enfermería después de la notificación de cada adversidad. Conclusión: La cultura de seguridad aún no está en el estándar ideal, siendo necesario el cumplimiento de una educación permanente y una evaluación frecuente de los servicios prestados relacionados con la seguridad del paciente con câncer.

Descriptores: Seguridad del Paciente; Enfermería; Cáncer. 


\section{INTRODUÇÃO}

A segurança do paciente passou a ser conhecida e discutida a partir da publicação, em 1999, do relatório “To err is Human: Building a safer health care system" (Errar é Humano: Construindo um sistema de saúde mais seguro), do Institute of Medicine, dos Estados Unidos da América (EUA), o qual apresentou números alarmantes a respeito de erros relacionados aos cuidados prestados por profissionais de saúde. De modo que a amplitude da ocorrência dos eventos adversos impulsionou entidades nacionais e internacionais a estabelecer metas voltadas à segurança do paciente (1).

Em 2009, a Organização Mundial de Saúde (OMS) conceituou a segurança do paciente "são ações que minimizem os riscos associados às práticas de assistência à saúde, visando prevenir e reduzir ao máximo os resultados decorrentes dos erros relacionados ao cuidado". Com isso, foram identificados os erros mais frequentes provocados pelos profissionais de saúde que abrange o doente errado; medicamento errado; dose/frequência errada; forma farmacêutica ou apresentação errada; via errada; quantidade errada; rótulo/instrução de administração errada; contraindicação; armazenamento errado; dose ou medicamento omitido; medicamento fora da validade. Além do erro no preenchimento de informações básicas como as características básicas da vítima (faixa etária e sexo), ou o desfecho do caso $(1,2)$.

No Brasil, a segurança do paciente passou a ser discutida a partir da instituição da Portaria MS/GM no 529 de 01 de abril de 2013, a qual iniciou o Programa Nacional de Segurança do Paciente (PNSP), que deveria ser implantado em todos os estabelecimentos de saúde do território nacional, de modo a colaborar com a qualidade da assistência em saúde(3). No mesmo ano, foi instituída a Resolução - RDC no 36 da Agência Nacional de Vigilância Sanitária (ANVISA), a qual instaurou ações para segurança do paciente, sendo elas: criação do Núcleo de Segurança do Paciente (NSP); Plano de Segurança do Paciente (PSP); e Vigilância, Monitoramento e Notificação de Eventos Adversos (4).

A segurança do paciente começou a ser tratada como tema relevante a ser discutido e implementado nas diversas esferas de atendimento dos serviços de saúde, tendo destaque a assistência hospitalar ao paciente em especial o oncológico. Isto devido o crescente aumento de pacientes acometidos pelo câncer e consequentemente aumentando o tempo de internação, os procedimentos hospitalares e a intervenção da equipe de saúde no processo de diagnóstico e tratamento (5).

Segundo o Instituto Nacional de Câncer José Alencar Gomes da Silva (INCA), a estimativa para o Brasil, no biênio 2018/2019, a ocorrência de 600 mil casos novos de câncer, para cada ano. Excetuando-se o câncer de pele não melanoma (cerca de 170 mil casos novos), ocorrerão 420 mil casos novos de câncer. Nos anos de 2016 e 2017, é da incidência de cerca de 600 mil casos novos de câncer. Somente em 2011, cerca de 14.395 internações de pacientes oncológicos foram realizadas, com tempo de internação médio de 7,82 dias. Tais internações decorrem em procedimentos invasivos, cirúrgicos e clínicos que oferecem diversos riscos de erros e eventos adversos associados aos cuidados em saúde $(6,7)$.

Para que a assistência em saúde seja oferecida de forma adequada ao paciente acometido por câncer, o enfermeiro necessita possui conhecimento técnico-científico adequado de modo a ofertar cuidados hospitalares complexos de acordo com as necessidades afetadas em decorrência da patologia. Assim, o enfermeiro está diretamente ligado a segurança do paciente oncológico, devido às ocupações inerentes à categoria profissional (8).

A enfermagem cada vez mais têm buscado estratégias para promover a melhoria da assistência de enfermagem ao paciente oncológico. Tal assistência requer conhecimentos específicos, pelo fato de o câncer se configurar como uma patologia que necessita de cuidados complexos e oferece riscos e agravos durante o tratamento. Assim, o enfermeiro que presta cuidados assistenciais ao paciente com câncer, deve oferecer um cuidado em saúde com qualidade e segurança adequados, de forma a minimizar possiveis erros e complicações (9). Pelo exposto, torna-se relevante para o âmbito da segurança do paciente oncológico e da enfermagem, o seguinte questionamento: Quais as práticas adotadas por enfermeiros para a segurança de pacientes oncológicos?

O objetivo deste artigo é avaliar as práticas adotadas por enfermeiros para a segurança dos pacientes oncológicos.

\section{METODOLOGIA}

\section{Tipo de estudo}

Estudo exploratório, descritivo, de abordagem quantitativa.

\section{Local do estudo}

O estudo foi desenvolvido no Hospital Ophir Loyola, que é o hospital referência da Região Norte em tratamento oncológico. Critérios de inclusão: participaram enfermeiros que atuam diretamente na assistência ao paciente e que trabalham nos turnos matutino e vespertino. Critérios de exclusão: enfermeiros que atuam exclusivamente na gerência e os que estavam de férias no momento do estudo.

\section{População e amostra}

A população total de profissionais foi de 58 enfermeiros, sendo que a amostra da pesquisa foi de 51 enfermeiros no 
exercício das atividades de enfermagem alocados nos setores de Clínica Cirúrgica, Primeiro Departamento de Câncer, Segundo Departamento de Câncer, Centro de Terapia Intensiva (CTI) e ambulatório. Período da coleta de dados ocorreu de janeiro a março de 2018.

\section{Procedimentos de coleta}

A abordagem dos participantes foi realizada em seu ambiente de trabalho, sendo a escolha destes de forma aleatória, respeitando a disponibilidade para responder ao questionário, aceitação de participação da pesquisa e assinatura do Termo de Consentimento Livre e Esclarecido (TCLE), sendo esta abordagem realizada em um setor por vez. Inicialmente os pesquisadores se apresentavam, explanando acerca da pesquisa e de sua importância e convidandoos a participar. Os participantes que concordaram eram acompanhados a um ambiente reservado, e após a leitura e aceite do TCLE, respondiam ao questionário.

\section{Instrumento de coleta de dados}

A coleta se deu por meio da aplicação de um questionário intitulado "Pesquisa sobre Segurança do Paciente em Hospitais" (HSOPSC), da "Agency Healthcare Research and Quality" dos EUA, que tem como objetivo avaliar as múltiplas dimensões da cultura de segurança do paciente e questiona a opinião de seus respondentes sobre pontochaves relacionados à segurança, valores, crenças e normas da organização, notificação de eventos adversos, comunicação, liderança e gestão. As respostas obtidas foram organizadas em consonância com as variáveis abordadas por testes paramétricos para desenvolver a conclusão acerca da avaliação das práticas abordadas sobre a segurança dos pacientes oncológicos.

\section{Procedimentos de análise}

A análise ocorreu por intermédio da estatística descritiva, as variáveis na pesquisa incluíram o gênero, grau de instrução dos participantes, carga horária semanal, tempo de trabalho no hospital e a área/unidade de trabalho dos participantes. Além disso, foi aplicada a Escala de Likert para avaliar o nível de concordância dos participantes sobre as respostas dadas ao serem questionados sobre a aplicação de medidas de segurança do paciente no hospital. A avaliação abrange com respostas variando de muito ruim a excelente.

\section{Procedimentos éticos}

Após a autorização da realização da pesquisa no Hospital, da aprovação do Comitê de Ética e da assinatura do Termo de Consentimento Livre Esclarecido- TCLE o questionário foi aplicado. Sendo todos codificados com letras e números para evitar a identificação dos enfermeiros, resguardandose 0 anonimato. $O$ projeto foi aprovado pelo Comitê de Ética em Pesquisa (CEP) da Escola de Enfermagem "Magalhães Barata" da Universidade do Estado do Pará (CAAE 73031417.1.0000.5170) e o CEP do Hospital Ophir Loyola (CAAE 73031417.1.3001.5550).

\section{RESULTADOS}

Pode-se constatar na tabela 1 a seguir, com a utilização das variáveis abordadas, a predominância do gênero feminino nos cuidados de pacientes oncológicos, sendo o total de 44 (86,3\%) profissionais. Quanto ao grau de instrução, a PósGraduação Lato sensu (Especialização) prevaleceu com 43 (84,3\%) e com Pós-Graduação Stricto sensu 3 (5,9\%). Em relação ao tempo de trabalho no hospital, 15 (29,4\%) enfermeiros afirmaram que trabalham no hospital de 1 a 5 anos. Além disso, à carga horária semanal relatada pelos participantes, 26 (51,0\%), está entre 20 a 39 horas semanais. Ademais, observa-se que a maior parte dos participantes trabalham em unidades de cirúrgicas 27,5\% (14) e unidade de terapia intensiva $27,5 \%$ (14).

Tabela 1: Perfil dos enfermeiros do Hospital Ophir Loyola. Belém-PA. 2018.

\begin{tabular}{|c|c|c|}
\hline Gênero & $\mathrm{N}$ & $\%$ \\
\hline Feminino & 44 & 86,3 \\
\hline Masculino & 7 & 13,7 \\
\hline TOTAL & 51 & 100 \\
\hline Grau de Instrução & $\mathrm{N}$ & $\%$ \\
\hline $\begin{array}{l}\text { Ensino Superior Com- } \\
\text { pleto }\end{array}$ & 5 & 9,8 \\
\hline $\begin{array}{l}\text { Pós-graduação (Nivel } \\
\text { Especialização) }\end{array}$ & 43 & 84,3 \\
\hline $\begin{array}{l}\text { Pós-graduação (Nivel } \\
\text { Mestrado ou Douto- } \\
\text { rado) }\end{array}$ & 3 & 5,9 \\
\hline TOTAL & 51 & 100 \\
\hline $\begin{array}{c}\text { Tempo de Trabalho no } \\
\text { Hospital }\end{array}$ & $\mathrm{N}$ & $\%$ \\
\hline Menos de 1 ano & 11 & 21,5 \\
\hline la 5 anos & 15 & 29,4 \\
\hline 6 a 10 anos & 10 & 19,6 \\
\hline 11 a 15 anos & 8 & 15,7 \\
\hline 16 a 20 anos & 5 & 9,8 \\
\hline 21 anos ou mais & 2 & 3.9 \\
\hline TOTAL & 51 & 100 \\
\hline Carga Horária Semanal & N & $\%$ \\
\hline Menos de 20 horas & 4 & 7,8 \\
\hline 20 a 39 horas & 26 & 51 \\
\hline 40 a 59 horas & 16 & 31,4 \\
\hline 60 a 79 horas & 5 & 9,8 \\
\hline
\end{tabular}

80 a 99 horas 


\begin{tabular}{ccc}
$\begin{array}{c}\text { 100 horas ou mais } \\
\text { TOTAL }\end{array}$ & - & - \\
$\begin{array}{c}\text { Principal área/unidade de } \\
\text { trabalho }\end{array}$ & 51 & 100 \\
\hline $\begin{array}{c}\text { Cirurgia } \\
\text { UTI }\end{array}$ & 14 & $\%$ \\
\hline $\begin{array}{c}\text { Clinica (não cirúrgica) } \\
\text { Setor de Emergência }\end{array}$ & 14 & 27,5 \\
$\begin{array}{c}\text { Diversas unidades do } \\
\text { hospital/nenhuma } \\
\text { unidade especifica }\end{array}$ & 2 & 27,5 \\
\hline Outras & 2 & 9,8 \\
\hline $\begin{array}{c}\text { TOTAL } \\
\text { Tempo de trabalho na } \\
\text { atual área/unidade do } \\
\text { hospital }\end{array}$ & 14 & 3,9 \\
\hline Menos de l ano & 51 & 3,9 \\
\hline 1 a 5 anos & $\mathrm{N}$ & 27,4 \\
\hline 6 a 10 anos & 17 & 100 \\
11 a 15 anos & 18 & $\%$ \\
16 a 20 anos & 12 & 33,3 \\
21 anos ou mais & 4 & 35,3 \\
\hline TOTAL & - & 23,5 \\
\hline
\end{tabular}

Os participantes da pesquisa avaliaram a segurança do paciente na sua área de acordo com uma nota baseado na Escala de Likert com a estratificação de muito ruim a excelente. Logo, dos 51 enfermeiros, 25 (43,1\%) avaliaram a segurança do paciente como regular.

Gráfico 1: Avaliação da segurança do paciente no hospital. Belém-PA. 2018.

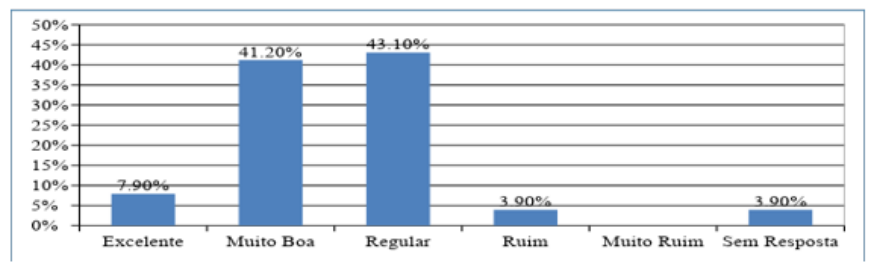

Aos participantes da pesquisa também foi perguntado a quantidade de notificações de eventos preenchidas nos últimos 12 meses. Constatou-se que $22(43,1 \%)$ profissionais não relataram nenhuma notificação. Já 4 (7,9\%) dos profissionais alegaram que ocorreu de 11 a 20 eventos que prejudicaram de forma direta ou indireta o bem-estar do paciente oncológico.

\section{DISCUSSÃO}

Na pesquisa o gênero feminino mostrou-se predominante como força de trabalhos nos setores supracitados. Contudo, o gênero não é um indicativo de avaliação para definir competência no que determina a segurança do paciente. Este maior número é justificável devido a maior parte dos profissionais atuantes de enfermagem serem do sexo feminino(10).

Entretanto, ao relacionar cuidados com o paciente ao grau de instrução, pode-se verificar um hospital com corpo profissional bastante capacitado. De modo que mais de 90\% dos enfermeiros que atuam nos setores possuem PósGraduação Lato sensu, de forma que a elaboração de planos no o cuidado do paciente proporciona maior qualidade técnica. $\mathrm{O}$ que se justifica pela porcentagem de relatos de Eventos notificados ocorridos durante um ano. Conforme os resultados apresentados 22 (43,1\%) dos enfermeiros não relataram nenhuma notificação(10)

$\mathrm{Na}$ análise da carga horária semanal de trabalho, predomina o período de 20 a 39 horas semanais (51,0\%). É sabido que longas jornadas de trabalho causam exaustão ao profissional, estresse e por vezes, levam à ocorrência de falhas na assistência e eventos adversos. Entretanto, na pesquisa verifica-se que a maior parte dos participantes possui uma carga horária de trabalho adequada, a qual evita que ocorram erros na assistência por cansaço, fadiga, dentre outros ${ }^{(10)}$.

Sobre as medidas adotadas para segurança dos pacientes oncológicos, baseados nas respostas no questionário aplicado, mostrou-se positiva em apresentar respostas fundamentais para uma assistência digna ao paciente oncológico. Entretanto, ocorreu contradições quando os profissionais não relataram aos pesquisadores os erros mais frequentes, gravidade, ou as respectivas ações implementadas para a prevenção(11).

Com relação à frequência de eventos relatados na área de trabalho, predominando as respostas positivas $(50,4 \%)$. Estas respostas podem indicar que a maioria dos profissionais afirmam realizar as notificações dos erros/eventos adversos. No entanto, ao se comparar estas respostas com o número de eventos notificados por participante, observa-se que a maioria dos entrevistados não realizaram nenhuma notificação no último ano sendo 43,1\%. Estes dados são contraditórios, pois, é observado que apesar de os profissionais afirmarem que as notificações são realizadas, o número destas relatadas por profissional é pequeno. Existe a possibilidade de subnotificações de eventos, as quais devem ser combatidas, pois pode existir o risco de profissionais estarem cometendo os mesmos erros inúmeras vezes, causando eventuais transtornos na saúde do paciente oncológico(11-13).

Além disso, foi detectado disparidade de opiniões entre os enfermeiros, uma vez que 22 (41,2\%) consideram a segurança do paciente como muito boa ou excelente e 25 $(43,1 \%)$ consideravam a mesma como regular. Considera-se que os profissionais que optaram pela classificação de nota "regular" julgam que o hospital está caminhando para uma segurança do paciente efetiva, mas ainda está em processo 
de implantação de medidas de segurança em alguns setores, e em etapa de construção de uma cultura de segurança entre a equipe, ainda não alcançando o nível desejado. Esta opinião é vista como positiva, uma vez que, é benéfico ao serviço hospitalar que os enfermeiros sejam capazes de reconhecer que há falhas, verificar quais são elas e buscar corrigi-las ${ }^{(14-16)}$.

\section{Limitações do estudo}

No decorrer da coleta de dados, foram observadas fragilidades no processo de aplicação do questionário, a saber: a recusa por alguns enfermeiros em participar da pesquisa devido o extenso questionário, o preenchimento incompleto do questionário por alguns participantes devido não considerarem algumas questões aplicáveis ao seu local de trabalho; verificando-se a necessidade de realizar avaliações em um curto período de tempo, de modo que o profissional não considere a participação na pesquisa como prejuízo para suas atividades. Também é necessária a avaliação das especificidades de cada setor, para que estes sejam avaliados com maior eficácia. Ressalta-se a dificuldade encontrada em realizar a aplicação do instrumento de coleta de dados para um público maior, devido à grande extensão do questionário e as muitas atribuições do enfermeiro em oncologia.

\section{Contribuições do estudo para a prática}

A realização deste estudo permitiu visualizar as mais variadas dimensões da segurança do paciente no hospital oncológico, bem como conhecer o perfil dos enfermeiros assistenciais e suas opiniões em relação a segurança do paciente oncológico em sua unidade de trabalho. Também foi possivel conhecer as práticas dos enfermeiros sobre as medidas de segurança ao paciente oncológico.

Os resultados da pesquisa demonstraram que grande parte dos participantes considera a segurança do paciente em seu ambiente de trabalho como boa. No entanto, são apresentadas contradições quanto a esta afirmativa no decorrer dos resultados obtidos, como o baixo percentual de notificações relatado, sendo sugerida a existência de subnotificação.

Em comparativo, o maior percentual dos entrevistados considerou a nota de segurança do paciente como regular, havendo um reconhecimento por estes de que ainda há falhas a serem observadas e corrigidas, bem como a necessidade de melhorias na implantação de protocolos na prática clínica, notificação adequada de eventos e avaliação periódica dos serviços e da segurança no cuidado.

\section{CONSIDERAÇÕES FINAIS}

Foi possivel avaliar as práticas adotadas por enfermeiros para a segurança dos pacientes oncológicos. Observouse que na sua maioria, os enfermeiros participantes da pesquisa buscam proporcionar a seus pacientes por meio de sua assistência um cuidado seguro e livre de danos. É reconhecido por estes participantes que ainda há barreiras a serem enfrentadas e dificuldades a serem superadas, mas os profissionais demonstram-se em geral, empenhados em prestar aos pacientes oncológicos um cuidado qualificado.

Ressalta-se ainda a subnotificação, problema este diagnosticado pelos próprios enfermeiros no momento da aplicação do instrumento de coleta de dados. Esta subnotificação ocorre por diversos fatores, seja por desconhecimento de sua importância, por falta de acesso aos documentos necessários para realizá-la ou até mesmo pelo medo de repreensões. No entanto, a notificação de erros e eventos adversos possui caráter decisivo no processo de avaliação das falhas e busca por avanços para um cuidado cada vez mais seguro.

Assim, pode-se compreender que a segurança do paciente oncológico é extremamente relevante, visto que estes pacientes, estando com a saúde fragilizada, necessitam de cuidados redobrados, e estão mais propensos a sofrer com erros e eventos adversos devido sua alta complexidade de assistência. Portanto, cabe a cada enfermeiro avaliar sua assistência prestada e buscar, cada vez mais um cuidado livre de danos.

E esta busca deve ser apoiada diretamente pelos demais membros da equipe multiprofissional e pelos gestores do hospital, proporcionando a ambiência e os recursos necessários para que o paciente oncológico seja tratado com as medidas de segurança adequadas, recebendo um tratamento de qualidade e livre de erros e eventos adversos.

\section{Contribuição dos autores}

Concepção e/ou desenho, análise e interpretação dos dados, redação do artigo, revisão crítica, revisão final: Sara Maria da Costa Negrão,Mayara Nicodemos da Conceição, Manoel Junior Ferreira Mendes, Jeferson Santos Araújo, Ingrid Magali Souza Pimentel, Mary Elizabeth 


\section{REFERÊNCIAS}

1. Souza CS, Tomaschewski-Barlem JG, Dalmolin GL, Silva TL, Neutzling BRS, Zugno RM Estratégias fortalecedoras da cultura de segurança. Rev enferm UERJ.2019; 27: e 38670.

2. Mitchell R. Using the WHO International Classification of patient safety framework toidentify incident characteristics and contributing factors for medical orsurgical complication deaths. Applied Ergonomics, 2019; 82: e 102920.

3. Adamy EK. Reflexão acerca da interface entre a segurança do paciente e o processo de enfermagem. Rev Enferm Health Care [Online]. 2018;7(1):272-78.

4. Maia CS. Notificações de eventos adversos relacionados com a assistência à saúde que levaram a óbitos no Brasil, 2014-2016. Epidemiol. Serv. Saude.2018;27(2): e 2017320.

5. Aires RSP. Segurança do Paciente na assistência à saúde. Blucher Education Proceedings.2017:2(1):198 - 204.

6. Paixão DPSS. Adesão aos protocolos de segurança do paciente em unidades de pronto atendimento. Rev. Bras Enferm.2018;71(1):622-629.

7. Silva MEB, Torres QSN, Silva TB, Araújo CS, Alves TL. Práticas Integrativas e Vivências em Arteterapia no Atendimento a Pacientes Oncológicos em Hospital Terciário. Revist. Port. Saúde e Sociedade.2018:3(1):721-31.

8. Instituto Nacional de Câncer José Alencar Gomes da Silva-INCA. Coordenação de Prevenção e Vigilância. Dados consolidados. Rio de Janeiro: INCA; 2018.

9. Instituto Nacional de Câncer José Alencar Gomes da Silva. Coordenação de ensino, serviço de educação e informação. ABC do Câncer: Abordagem Básica para o controle do Câncer. 3ạ edição revista e atualizada.Rio de janeiro: INCA;2017.
10. Sandro Rogério Almeida Matos Junior; Samara Stephanny Morais Santos Matos. Assistência de enfermagem em emergências oncológicas: uma revisão integrativa da literatura no periodo de 2008 a 2016. Caderno de Graduação Ciências Biológicas e de Saúde Unit. 2018; 4(3):105-12.

11. Marques J. et al. Cultura de Segurança e o Processo de Comunicação entre Membros da Equipe de Enfermagem. Revista enfermagem atual. 2018;86(24).

12. Notaro KAM. et al. Cultura de segurança da equipe multiprofissional em Unidades de Terapia Intensiva Neonatal de hospitais públicos. Rev Lat-Americana de Enferm. 2019;27: e3167.

13. Ortega DB; D'Innocenzo M; Silva LM, Bohomol E. Análise de eventos adversos em pacientes internados em unidade de terapia intensiva. Acta Paul Enferm. 2017;30(2):168-73.

14. Neto ALS; Lima AJ; Souza RC. A segurança do paciente no ensino profissionalizante: relato de uma campanha. Revista ELO - Diálogos em Extensão. 2018:07(2):44 - 48.

15. Figueiredo ML; D'Innocenzo M. Eventos adversos relacionados con las prácticas asistenciales: una revisión integradora. Enferm. glob. 2017;16(47):621-35

16. Andrade LEL. et al. Cultura de segurança do paciente em três hospitais brasileiros com diferentes tipos de gestão. Ciênc. saúde colet. 2018;23(1):161 - 172 . 